\title{
A systematic comparison of on-axis and off-axis transmission Kikuchi diffraction
}

\author{
Niessen, F.; Burrows, A.; Fanta, A. Bastos da Silva
}

Published in:

Ultramicroscopy

Link to article, DOI:

10.1016/j.ultramic.2017.12.017

Publication date:

2018

Document Version

Peer reviewed version

Link back to DTU Orbit

Citation (APA):

Niessen, F., Burrows, A., \& Fanta, A. B. D. S. (2018). A systematic comparison of on-axis and off-axis

transmission Kikuchi diffraction. Ultramicroscopy, 186, 158-170. https://doi.org/10.1016/j.ultramic.2017.12.017

\section{General rights}

Copyright and moral rights for the publications made accessible in the public portal are retained by the authors and/or other copyright owners and it is a condition of accessing publications that users recognise and abide by the legal requirements associated with these rights.

- Users may download and print one copy of any publication from the public portal for the purpose of private study or research.

- You may not further distribute the material or use it for any profit-making activity or commercial gain

- You may freely distribute the URL identifying the publication in the public portal

If you believe that this document breaches copyright please contact us providing details, and we will remove access to the work immediately and investigate your claim 


\title{
A systematic comparison of on-axis and off-axis transmission Kikuchi
} diffraction

\author{
F. Niessen ${ }^{1, a}{ }^{*}, A$. Burrows $^{2, b}$, A. Bastos da Silva Fanta ${ }^{2, c}$ \\ ${ }^{1}$ Danish Hydrocarbon Research and Technology Centre (DHRTC), Technical University of \\ Denmark (DTU), Elektrovej, 2800 Kgs. Lyngby, Denmark \\ ${ }^{2}$ Center for Electron Nanoscopy (CEN), Technical University of Denmark (DTU), Fysikvej, 2800 \\ Kgs. Lyngby, Denmark

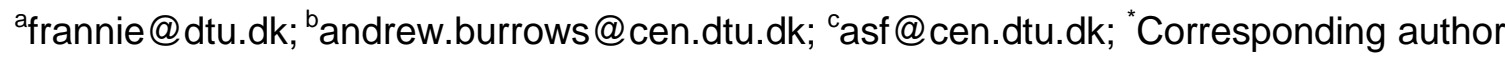 \\ Keywords: transmission Kikuchi diffraction, on-axis detector, orientation mapping, spatial \\ resolution, microstructure characterization
}

\begin{abstract}
The capabilities of the novel on-axis transmission Kikuchi diffraction (TKD) technique were explored in a systematic comparison with conventional off-axis TKD. The effect of experimental parameters on the appearance of on-axis and off-axis Kikuchi patterns was measured and discussed. In contrast to off-axis TKD, on-axis TKD is more sensitive to changes in beam current and beam energy and less sensitive to changes in working distance and detector distance. Moreover, on-axis TKD has a distinct advantage over off-axis TKD due to enhanced pattern intensity, which allows reduction of the beam current or an increase in the acquisition rate. The physical and effective spatial resolution were measured with detector-typical parameters. Even though the spatial resolution of both configurations did not differ significantly under test conditions, on-axis TKD enables measurement over large areas with the determined resolution, whereas offaxis TKD is more sensitive to beam drift. Band detection by the Hough-transform led to indexing of, on average, one additional Kikuchi band when measuring with on-axis TKD compared to offaxis TKD and operated more stable on on-axis patterns.
\end{abstract}

\section{Introduction}

It is half a decade since transmission Kikuchi diffraction (TKD) was introduced as a novel diffraction technique for the scanning electron microscope (SEM) [1,2]. The technique is capable of mapping the phases and orientations of nano-structured materials on a scale and resolution range between electron backscatter diffraction (EBSD) in the SEM and diffraction techniques in the transmission electron microscope (TEM). The technique consists of interaction of the scanning electron beam with an electron-transparent sample, which is tilted toward a conventional EBSDdetector to capture diffracted electrons. The captured diffraction patterns are Kikuchi-patterns, 
which arise from Bragg-diffraction of incoherently scattered electrons. The diffraction patterns obtained and the applied hardware are similar to those used in EBSD, which initially led to naming the technique transmission EBSD (t-EBSD) [3]. TKD enables orientation mapping with significant improvement in lateral spatial resolution compared to EBSD. Measurements on a Ni sample showed that the technique is capable of reaching a physical lateral spatial resolution of better than $10 \mathrm{~nm}$ [4].

It is apparent that TKD offers great benefits when orientation mapping and phase identification at the nanoscale over medium sized areas is required. The development of TKD occurred rapidly within a few years along with conventional EBSD hardware. In EBSD the sample is tilted approx. $70^{\circ}$ away from the incident beam to capture the maximum intensity of backscattered electrons forming the Kikuchi pattern. In TKD, Kikuchi patterns are formed by forward-scattered electrons for which the highest intensity occurs close to the incident beam, i.e. below the electron transparent sample. Acquisition of Kikuchi patterns in TKD with EBSD hardware thus occurs far away from the pattern center, and leads to acquisition of only limited diffracted intensity and severe gnomonic distortion of the Kikuchi pattern.

These limitations triggered the development of a new detector configuration by Fundenberger et al., which consists of a phosphor screen positioned below the sample normal to the incident beam, analogous to TEM [5]. As the acquisition occurs on the axis of the incident beam, TKD in this configuration is termed “on-axis” TKD, compared to conventional "off-axis” TKD [6].

Yuan et al. showed that the new configuration leads to high intensity and low gnomonic distortion of acquired Kikuchi patterns. Either the electron dose or the acquisition time could be lowered by a factor of 20 to yield the same pattern intensity as observed in off-axis TKD.[6] Brodu et al. demonstrated that, depending on the experimental parameters, a wide range of diffraction patterns such as diffraction spots, thin Kikuchi lines and bright or dark Kikuchi bands, are accessible with the on-axis TKD-detector. It is suggested that the sharp Kikuchi lines in on-axis TKD may enable high angular resolution measurements and even strain determination [7]. When using an on-axis detector, the Kikuchi pattern includes the transmitted beam, which leads to a bright spot in the center of the pattern. In a recent review by Sneddon et al. it was speculated that the bright spot may cause issues with indexing of relatively indistinct patterns or when high angular resolution is desired [8].

The current state of literature on the relatively young detector system is limited and requires further investigation to distinguish all the advantages and drawbacks of the systems. The present 
investigation therefore aims to compare on-axis and off-axis TKD to gain a better assessment of both techniques. The effect of different experimental parameters on the appearance of the Kikuchi pattern is reported and discussed. Furthermore the physical and effective spatial resolutions are explored when applying typical parameters for each detector. Finally, the robustness of band detection by the Hough transform for on-axis and off-axis Kikuchi patterns is tested and general, more practical, remarks on the new detector configuration are reported.

\section{Experimental procedure}

Before stating the details of the experimental procedures, the definitions for physical and effective spatial resolution should be established. The physical spatial resolution (PSR) is determined by the distance from a grain boundary in which the pattern of a neighboring grain is first observed. The effective spatial resolution (ESR) is defined by the distance from the grain boundary in which two overlapping patterns can be clearly distinguished by the indexing software. The prior is thus directly related to the electron interaction volume, while the latter is furthermore affected by the ability of software algorithms to deconvolute overlapping patterns. Consequently, the ESR is generally better than the PSR [9].

\subsection{Introduction to on-axis TKD}

As briefly outlined in the introduction, the on-axis detector configuration for TKD consists of a phosphor screen oriented normal to the incident beam, which is positioned below an electron transparent sample. Figure 1 shows a schematic of the on-axis and off-axis detector configurations. Physically speaking, the only difference of the two configurations is the angle $\alpha$, which is termed the intersection angle of the phosphor screen with the incident beam. The intersection angle has a magnitude of few degrees in the off-axis detector configuration and is close to $90^{\circ}$ in the case of the on-axis detector configuration. A low intersection angle (off-axis TKD) leads to high gnomonic distortion and little acquired diffraction intensity, whereas an intersection angle close to $90^{\circ}$ (onaxis TKD) has the opposite effect.

The detector distance to the sample in on-axis TKD is adjusted by either changing working distance, $d_{w}$, or the detector tilt angle, $\beta$, which leads to negligible gnomonic distortion of the Kikuchi pattern. Figure 1 demonstrates that there is far more room for changing the detector distance, $d_{d}$, with the on-axis detector configuration. Therefore full intersection with the Kikuchi 


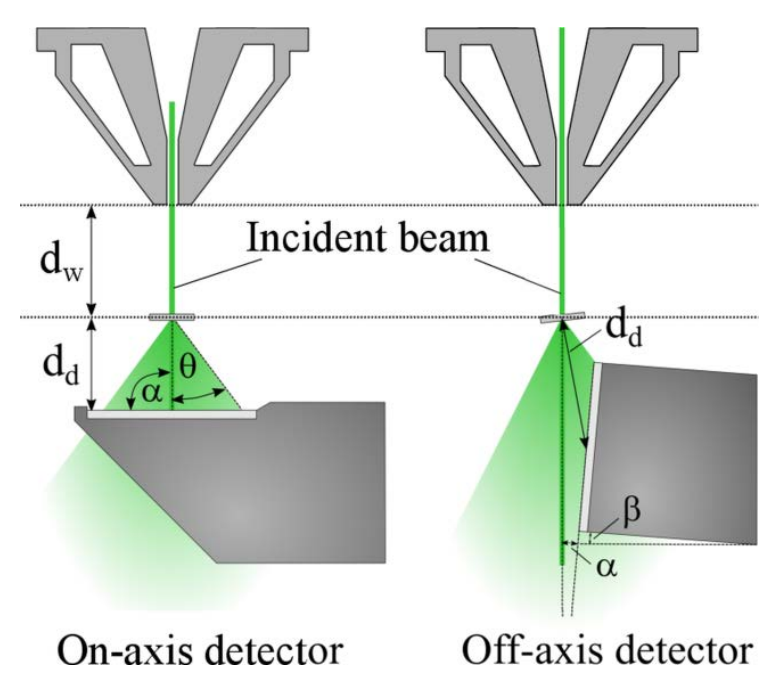

Figure 1 - Schematic of beam-specimen interaction and chamber geometry for the investigated detector systems showing the pole-piece, the incident and scattered electron beam (green), the sample and the detector heads, where $\mathrm{dw}$ is the working distance, dd is the detector distance, $\theta$ is the scattering semiangle, $\alpha$ the intersection angle and $\beta$ the detector tilt angle.

cone at its maximum intensity is possible, which leads to acquisition of a far greater part of the Kikuchi map compared to the off-axis detector. In on-axis TKD, the Kikuchi pattern also contains the transmitted beam intensity, which leads to a bright spot in the center of the pattern.

\subsection{Experimental setup}

The applied setup consisted of a Bruker e-Flash HD EBSD detector, installed in an FEI Nova NanoLab 600 SEM. The detector can be positioned with varying distance and tilt toward the sample and is used with interchangeable phosphor screen assemblies in vertical (off-axis) and horizontal (on-axis) orientation relative to the insertion axis of the detector. The on-axis OPTIMUS ${ }^{\mathrm{TM}}$ TKD detector head comprises a horizontally positioned scintillator, which directs the light from the phosphor screen toward the camera with help of a $45^{\circ}$ inclined reflection mirror. The detector is additionally equipped with diodes for bright- and dark-field imaging. A more detailed description of the detector head assembly can be found in Ref. [5]. Figure 2 shows both detector configurations in operation with $-20^{\circ}$ sample tilt in off-axis TKD, and no sample tilt in the case of on-axis TKD.

\subsection{Studied materials}

Two materials were the subject of the current study. For the parameter study and the study on the PSR it was found important to analyze a well-known material with low defect density, no strong 


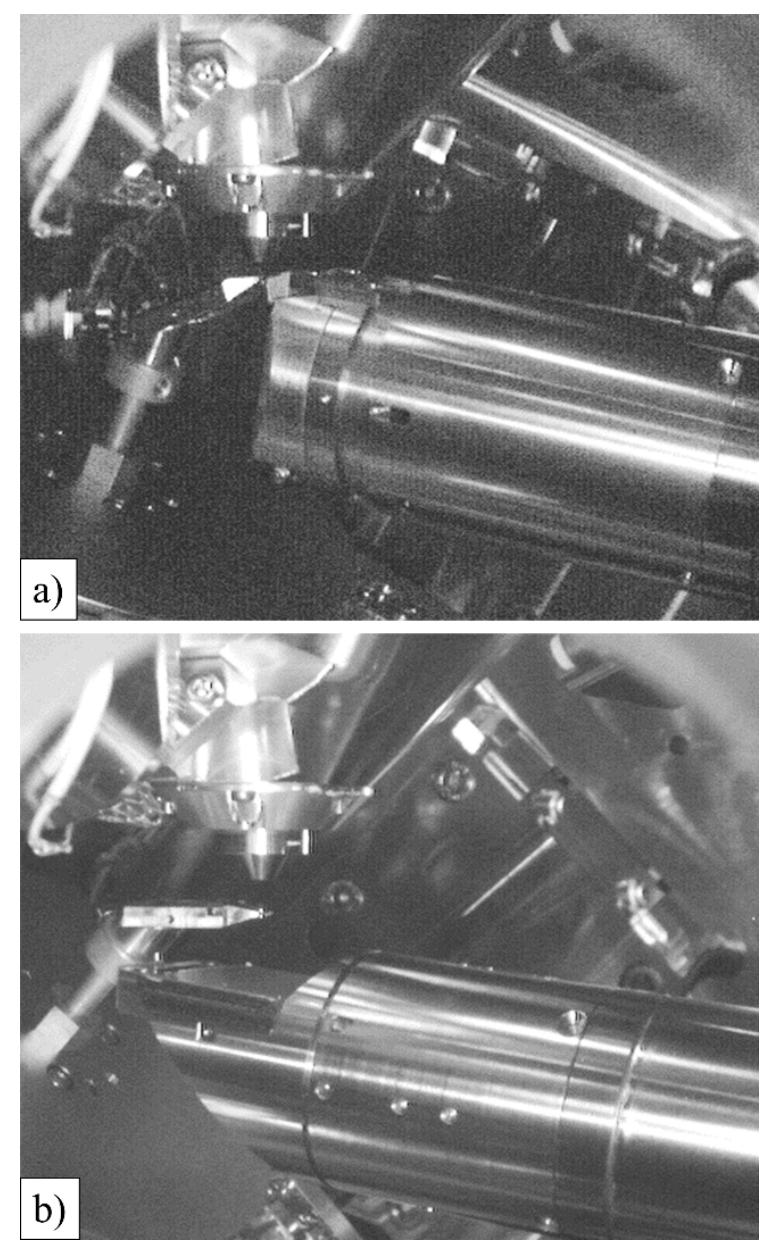

Figure 2a): Chamber geometry for TKD with the conventional off-axis detector configuration and $-20^{\circ}$ sample tilt; b): Chamber geometry with an on-axis detector configuration and no specimen tilt

substructure, large grains and well-defined grain boundaries. Thus an AISI 316L austenitic stainless steel was chosen. The material was received as sheet material of $1 \mathrm{~mm}$ thickness in bright cold rolled finished (2B) condition, with the composition given in Table 1.

The material was annealed at $700{ }^{\circ} \mathrm{C}$ for $2 \mathrm{~h}$ under continuous Ar-flow and was subsequently ground to approx. $100 \mu \mathrm{m}$ thickness. The thin-foil was produced by electrolytic twin-jet polishing with $10 \%$ perchloric acid at $-20{ }^{\circ} \mathrm{C}$. Prior to investigation, the specimen was plasma-cleaned. The thickness of the sites of interest investigated was determined in a TEM using the Electron energy loss spectroscopy (EELS) log-ratio technique [10]. The mean free path, $\lambda$, was approximated with

Table 1 - Chemical composition of the investigated AISI 316L austenitic steel determined with optical emission spectroscopy (OES) and balanced with Fe (wt.\%)

\begin{tabular}{c|c|c|c|c|c|c|c}
$\mathrm{Fe}$ & $\mathrm{Cr}$ & $\mathrm{Ni}$ & $\mathrm{Mo}$ & $\mathrm{Mn}$ & $\mathrm{Si}$ & $\mathrm{C}$ & $\mathrm{N}$ \\
\hline \hline bal. & 16.8 & 10.4 & 2.1 & 0.9 & 0.5 & 0.02 & 0.05
\end{tabular}


the MFP Estimator script by Mitchell for $120 \mathrm{keV}$ beam energy and a collection semi angle $\beta$ of 30 $\operatorname{mrad}[10,11]$. The mean free path was calculated as $\lambda=67 \mathrm{~nm}$ and yielded a foil-thickness at the

sites of interest of $t=115 \pm 8 \mathrm{~nm}$. The bright-field image of the investigated microstructure is shown with the positions of the sites of interest in Figure 3a.

In order to analyze the ESR a nanocrystalline gold thin-film was chosen. The sample was
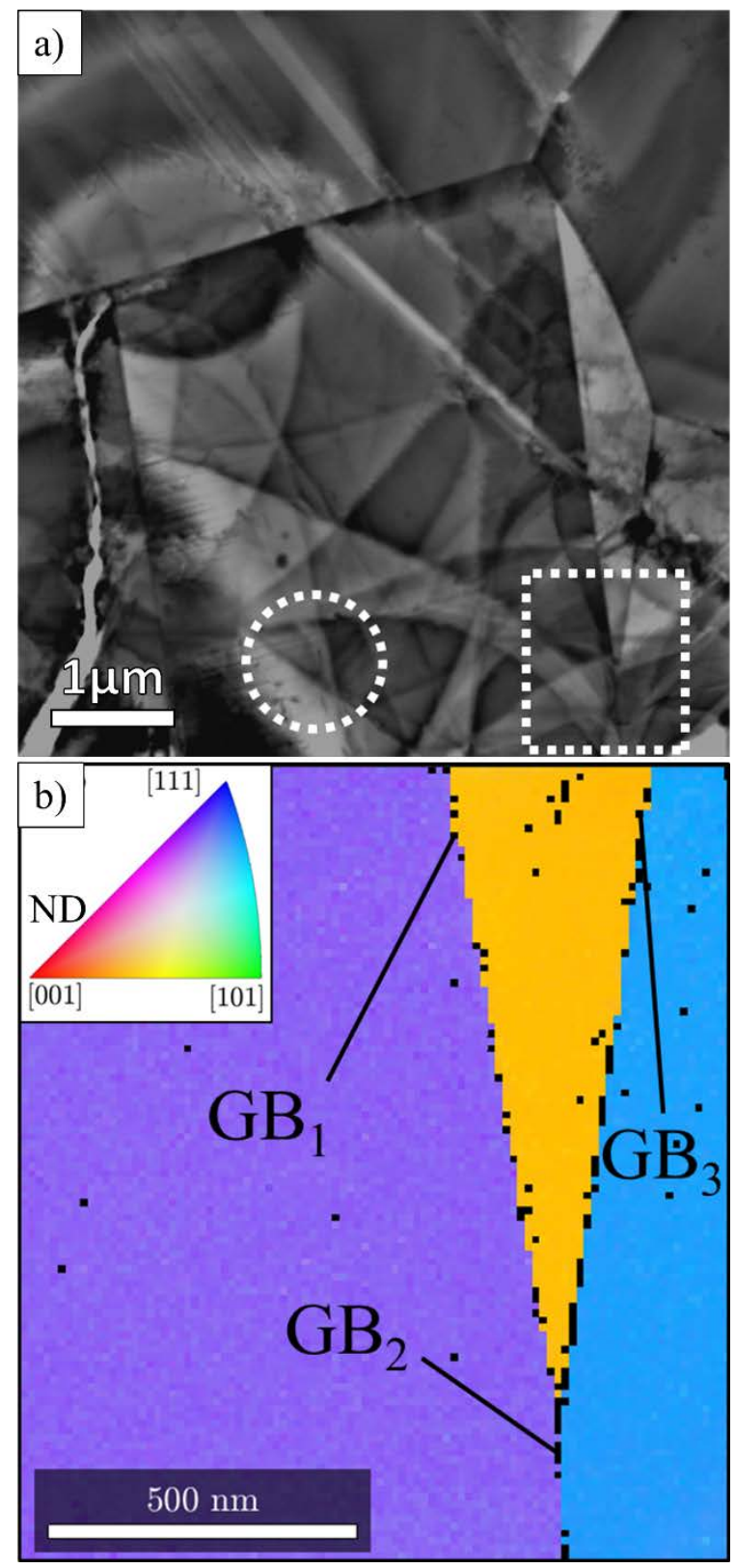

Figure 3a): Bright-field image of the AISI 316L thin foil, showing the area from which the Kikuchi patterns for the parameter study were taken (circle) and the area from which the PSR was measured (rectangle); The curved fringes in the image are bend contours, which are diffraction contrast effects arising from slight bending of the thin-foil; b): Inverse pole-figure map indicating the grain orientations at the HAGB's for measurement of the PSR (rectangle area in 3a) 
deposited by e-beam deposition at room temperature on $5 \mathrm{~nm}$ thin silicon nitride TEM windows. The total thickness of the gold film was approx. 15nm.

\subsection{Reference parameters and parameter study}

The first part of the experimental procedure consisted of a parameter study in which individual experimental parameters were varied from a set of reference parameters for each detector configuration. All Kikuchi patterns of the austenitic stainless steel sample were acquired from the encircled area shown in Figure 3a.

In order to carry out a systematic parameter study a set of reference parameters was defined for each detector configuration. The reference parameters are the result of iterative parameter optimization and represent typical parameters for the two detector configurations for obtaining high-resolution and high intensity Kikuchi patterns (Table 2). Relative to conventional parameters for TKD orientation mapping, the acquisition time was increased by a factor of approx. 10 to yield Kikuchi patterns of good quality for detailed analysis. The parameters were tuned to yield similar illumination in both detector configurations at identical pattern resolution. The working distance of the on-axis detector was increased to $6 \mathrm{~mm}$ in the parameter study to give a more even illumination of the detector; lower working distance led to constraints in space with the currently used sample holder. The increased illumination of the on-axis detector allowed acquisition with a smaller aperture and lower acquisition time. The beam current was accordingly reduced by $45 \%$ and the

Table 2- Reference parameters for investigations with off-axis and on-axis detector configurations SEM parameters

\begin{tabular}{|c|c|c|}
\hline & Off-axis detector & On-axis detector \\
\hline Beam energy [keV]: & 30 & 30 \\
\hline Spot size [1]: & 4 & 4 \\
\hline Aperture diameter $[\mu \mathrm{m}]:$ & 40 & 30 \\
\hline Beam current [nA]: & 1.5 & 0.83 \\
\hline Gun emission mode: & Analytical & Analytical \\
\hline \multicolumn{3}{|l|}{ Geometrical parameters } \\
\hline Sample tilt $\left[^{\circ}\right]:$ & -20 & 0 \\
\hline Working distance [mm]: & 3 & 6 \\
\hline Detector distance [mm]: & 13.5 & 13.9 \\
\hline Detector angle $\left[{ }^{\circ}\right]$ : & 8.7 & 5.8 \\
\hline \multicolumn{3}{|c|}{ Camera settings } \\
\hline Image resolution [pixels]: & $800 \times 600$ & $800 \times 600$ \\
\hline Exposure time [ms]: & 850 & 75 \\
\hline Gain [1]: & 0 & 0 \\
\hline
\end{tabular}


acquisition time was reduced by a factor of 11 . When severe over- or under-exposure of the camera was experienced, the acquisition time was adapted, which in that case is reported together with the respective results. The patterns for the qualitative parameter study were acquired in spot-mode, i.e. beam-blanking immediately before and after acquisition.

\subsection{Kikuchi band detection and robustness of indexing}

As previously discussed, the appearance of Kikuchi patterns in on-axis and off-axis is essentially different, which is expected to have consequences for band detection and robustness of indexing. Up to now, it has not been demonstrated whether the Kikuchi pattern obtained by on-axis TKD improves or deteriorates the indexing precision.

The number of correctly indexed Kikuchi bands from automatic, Hough-transform based, band detection and manually refined band detection are compared. The comparison should indicate how many bands generally are detected by the Hough transform from on-axis and off-axis Kikuchi patterns, and how many bands are wrongly or not detected. The parameters for the Hough-transform were kept as default and constant over the analysis. Two individual grains were analyzed for each detector configuration, where the pattern analysis was performed twice for both line detection methods. As the analysis involves manual refinement of band detection, the results should be considered as semi-quantitative.

\subsection{Spatial resolution}

The achievable PSR when conducting on-axis and off-axis TKD was investigated using detectortypical reference parameters to obtain high resolution Kikuchi patterns. As the PSR is directly dependent on the interaction volume formed by the electron beam and the sample, the different detector systems affect the measured PSR by enabling analysis with different beam energies, exposure time, sample tilt, and by acquiring signal from different source regions. The PSR was determined with the parameters stated in Table 2 by measuring the orientation across the three indicated high-angle grain boundaries (HAGBs) in Figure 3b.

Since the sets of reference parameters comprise different working distances, the working distance was varied in on-axis TKD to account for possible occurrence of beam-broadening. The line scans were carried out approximately perpendicular with respect to the grain boundary with a step size of $3 \mathrm{~nm}$. Exact parallel alignment of the grain boundaries with the primary electron beam in depth of the sample was not possible due to limited degrees of freedom of the microscope stage. 
The effect of minor misalignment on the spatial resolution measurements is however expected to have negligible effect since the majority of the signal contributing to the contrast of the Kikuchi pattern arises from the last few tens of nanometers of the exit surface [12]. It is furthermore expected that highly inclined boundaries would lead to a thick region of low band contrast at the boundary and an asymmetric line profile of the sum of correlation coefficients in the analysis of the PSR, which was not the case and thus suggests an approximately parallel orientation of the boundary with respect to the electron beam .

The PSR was determined by an image-correlation technique [13,14]. The Kikuchi patterns across a HAGB were compared to reference patterns of the individual grains far away from the HAGBs. An example of an overlay of two reference patterns is given in Figure 4a.

The comparison was based on analyzing the change in intensity of the grayscale pixels from cropped areas of Kikuchi patterns, which showed distinct features for both reference patterns. In this manner the correlation coefficient, $C_{\text {corr }}$, was determined by the following equation:

$$
C_{c o r r}=\frac{\sum_{m} \sum_{n}\left(I_{m, n}-\bar{I}\right)\left(I_{m, n}^{\text {ref }}-\overline{I^{r e f}}\right)}{\sqrt{\left(\sum_{m} \sum_{n}\left(I_{m, n}-\bar{I}\right)^{2}\right)\left(\sum_{m} \sum_{n}\left(I_{m, n}^{\text {ref }}-\overline{I^{r e f}}\right)^{2}\right)}}
$$

where $I$ is the intensity of the analyzed Kikuchi pattern and $I^{\text {ref }}$ the intensity of the reference Kikuchi pattern. Indices $m$ and $n$ denote the grayscale pixel positions and the overbars indicate average values. The analysis was carried out using the MATLAB Imaging Toolbox, where equation (2) is implemented as function corr2 [15]. The correlation coefficients were normalized to unity and smoothed by applying a moving average filter with a step-size of 5 , to reduce noise originating from the low signal-to-noise ratio of TKD patterns. All initially tested filtering methods induced slight broadening of the line profile, which was in the range of $5 \%$ for the moving average filter. The effect showed the same extent for off-axis and on-axis TKD patterns and is considered within the experimental accuracy of the method. The correlation coefficients of both grains were summed and normalized to unity (see the example in Figure 4b). Half of the full width at half maximum (FWHM) of the resulting Gaussian profile was taken as a measurement of the PSR. For each of the three measurements over a HAGB, 10 cropped pattern areas were analyzed, which resulted in 30 measurements of the PSR per measurement configuration.

The ESR of both detector systems in connection with the Bruker ESPRIT software was evaluated by measuring orientation maps on a nanocrystalline gold thin-film. The measurements were carried out with the same set of reference parameters as in all other measurements (Table 2), albeit with a lower pattern resolution of 320 x 240 pixels to reduce the effect of beam drift. No 
cleaning or smoothening algorithms were applied to the data sets, and a minimum of 5 Kikuchi bands were required for successful indexing.

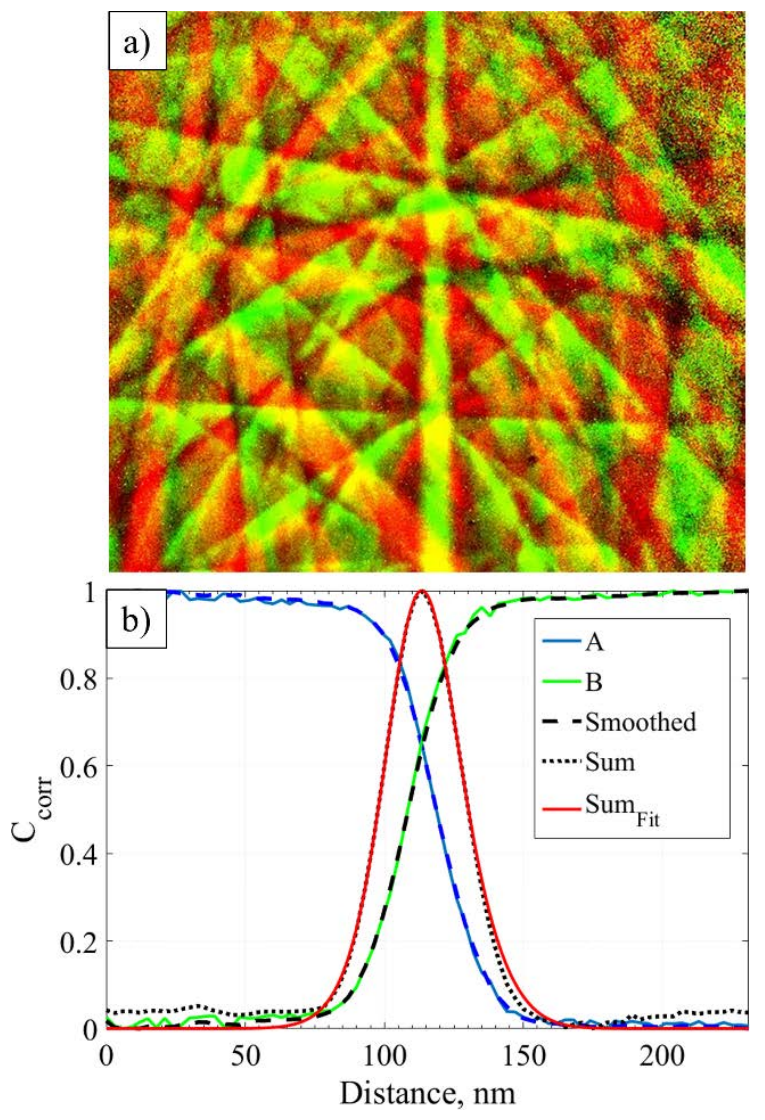

Figure 4a): Overlay of color-coded reference patterns of two grains (red and green); b): Example for the determination of the PSR by analysis of the correlation coefficient $C_{\text {corr }}$; The correlation of the patterns with the reference pattern of two grains $A$ and $B$ are plotted in the raw and smoothed version. The normalized sum of the two smoothed curves is fitted with a Gaussian profile, from which half the full width at half maximum represents the PSR.

\section{Experimental results}

\subsection{Experimental parameter study}

Experimental parameters were varied one by one from the set of reference parameters in Table 2. The Kikuchi patterns obtained with the reference parameters are given in the following subsection (Figure 5). All Kikuchi patterns which are formed with altered parameters are compared with the reference Kikuchi patterns.

\section{Reference parameters}

In Figure 5 the Kikuchi patterns acquired with the reference parameters in Table 2 are shown in real and Hough space, and are compared with their respective simulated patterns. The Kikuchi 
pattern simulation was carried out using the dynamical simulation model of the Bruker ESPRIT DynamicS software suite.

The Kikuchi pattern acquired with the off-axis detector system reveals great detail of the individual Kikuchi bands, but is subject to gnomonic distortion and only reveals a limited area of the Kikuchi map. The pattern obtained using the on-axis detector includes a larger area of the Kikuchi-map and thus more major zone axes. Although the pattern is disturbed by the transmitted electron beam in the center, which is surrounded by diffraction spots from coherent scattering, the Hough space does not seem to show any major disturbance by the acquisition of the transmitted beam. After subtraction of the area affected by the transmitted beam using conventional image processing software, the on-axis pattern yields approximately $70 \%$ additional area of the Kikuchimap compared to the off-axis Kikuchi pattern.
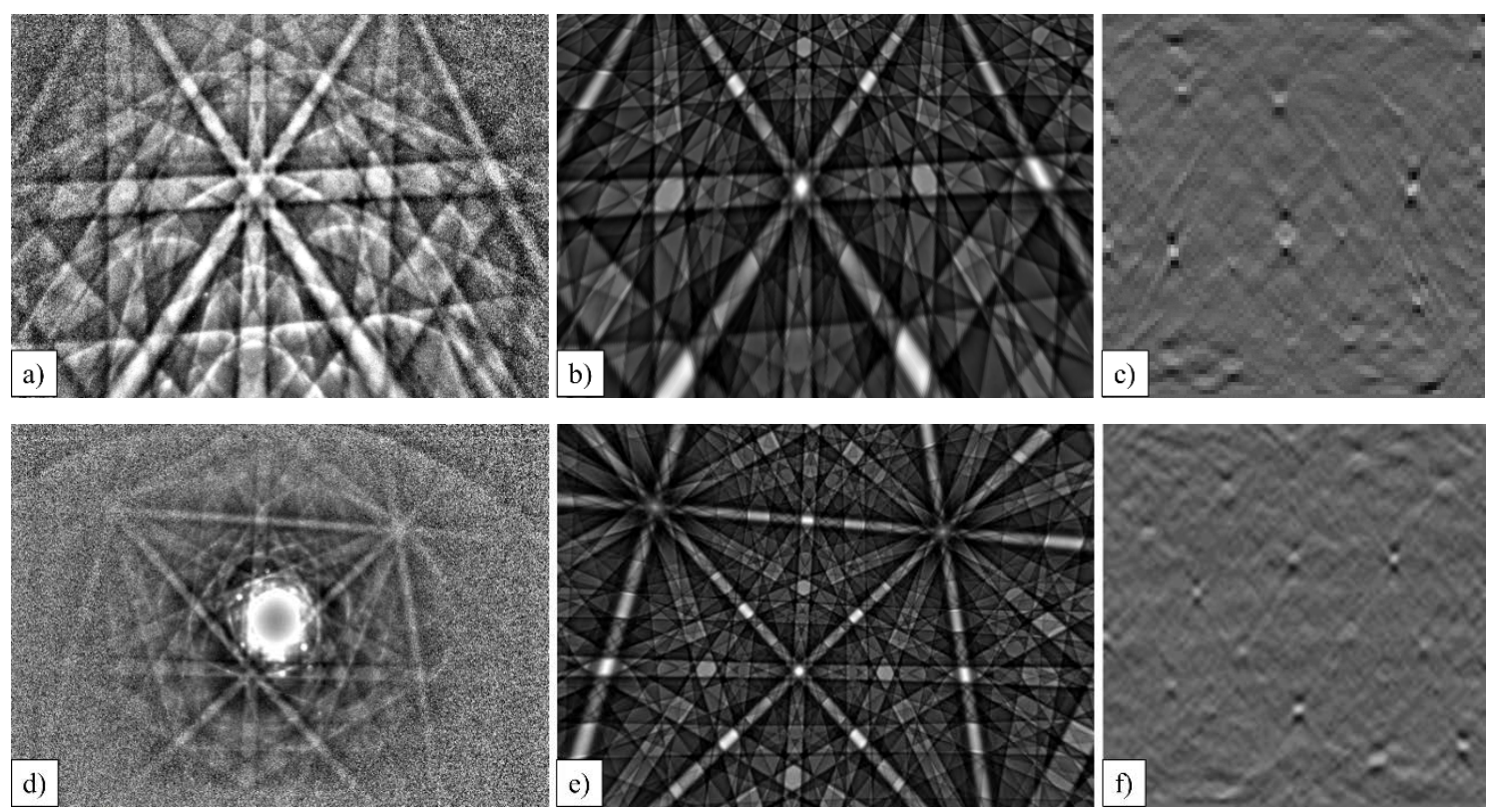

Figure 5 - Reference Kikuchi patterns acquired with the reference parameter sets in Table 2 using off-axis TKD (a) and on-axis TKD (d). The simulated patterns by dynamical simulation (b and e), and the Hough spaces (c and $f$ ) of the respective patterns.

\section{Detector distance}

The distance between detector and sample, $d_{d}$, (see Figure 1) was altered to investigate the effect on the Kikuchi pattern. Increasing the detector distance in off-axis TKD was carried out by retracting the camera. Progressive retraction led to intersection of the detector screen with the Kikuchi cone further away from the intensity maximum (c.f. Figure 1) and thus led to a gradual decrease in acquired intensity as well as a steady downward shift and magnification of the diffraction pattern. This effect is visible when comparing the Kikuchi patterns in Figure $5 \mathrm{a}\left(d_{d}=\right.$ $13.5 \mathrm{~mm})$, Figure $6 \mathrm{a}\left(d_{d}=18.5 \mathrm{~mm}\right)$ and Figure $6 \mathrm{~b}\left(d_{d}=23.5 \mathrm{~mm}\right)$. 

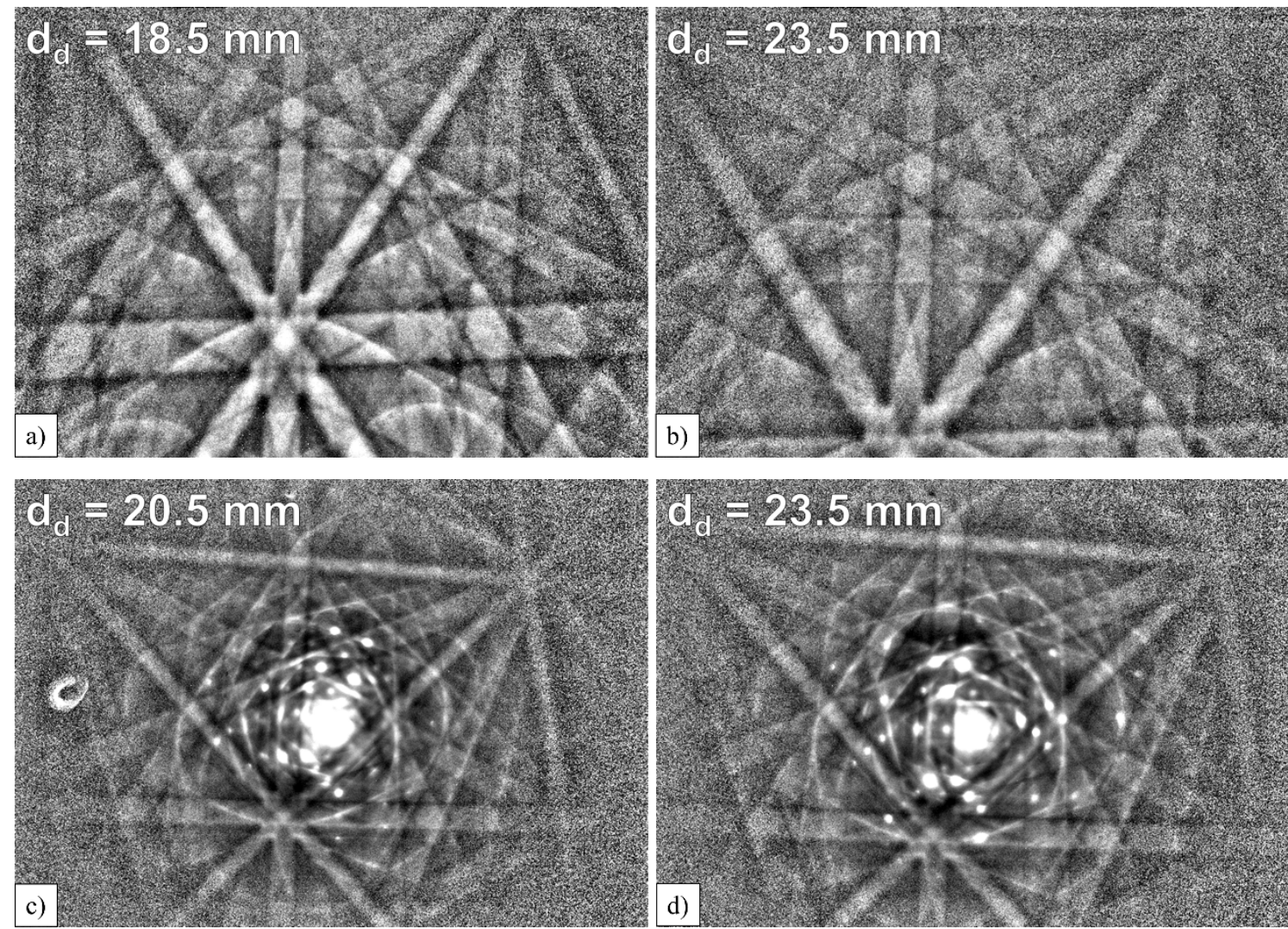

Figure 6 - Effect of changing the detector distance, $d_{d}$, on Kikuchi patterns compared to the reference patterns in Figure 5 in (a-b): off-axis TKD at a detector distance of 18.5 and $23.5 \mathrm{~mm}$ by variation in detector insertionlength; (c-d): on-axis TKD at a detector distance of 20.5 and $23.5 \mathrm{~mm}$ by changing the detector insertion-angle.

Variation of the detector distance in on-axis TKD was carried out by changing the detector tilt toward the sample. In this case the increase in detector distance led only to magnification of the Kikuchi-pattern without a pattern shift. The effect can be seen when comparing Figure $5 d\left(d_{d}=13.9\right.$ $\mathrm{mm})$, Figure $6 \mathrm{c}\left(d_{d}=20.5 \mathrm{~mm}\right)$ and Figure $6 \mathrm{~d}\left(d_{d}=23.5 \mathrm{~mm}\right)$. No noticeable gnomonic distortion is introduced by the detector tilt.

\section{Working distance}

Increasing the working distance, $d_{w}$, in off-axis TKD leads to a downward shift of the pattern center and Kikuchi pattern, and a loss of pattern contrast (Figure 5a, Figure 7a and Figure 7b). In the case of the on-axis detector it is straightforward to compensate changes in working distance by adapting the detector distance (tilting of the detector) to keep it constant. The change in working distance did not seem to noticeably affect the Kikuchi pattern (Figure 5d, Figure 7c and Figure 7d). As previously stated the default sample holder used did not allow optimal insertion of the detector at very low working distance. 

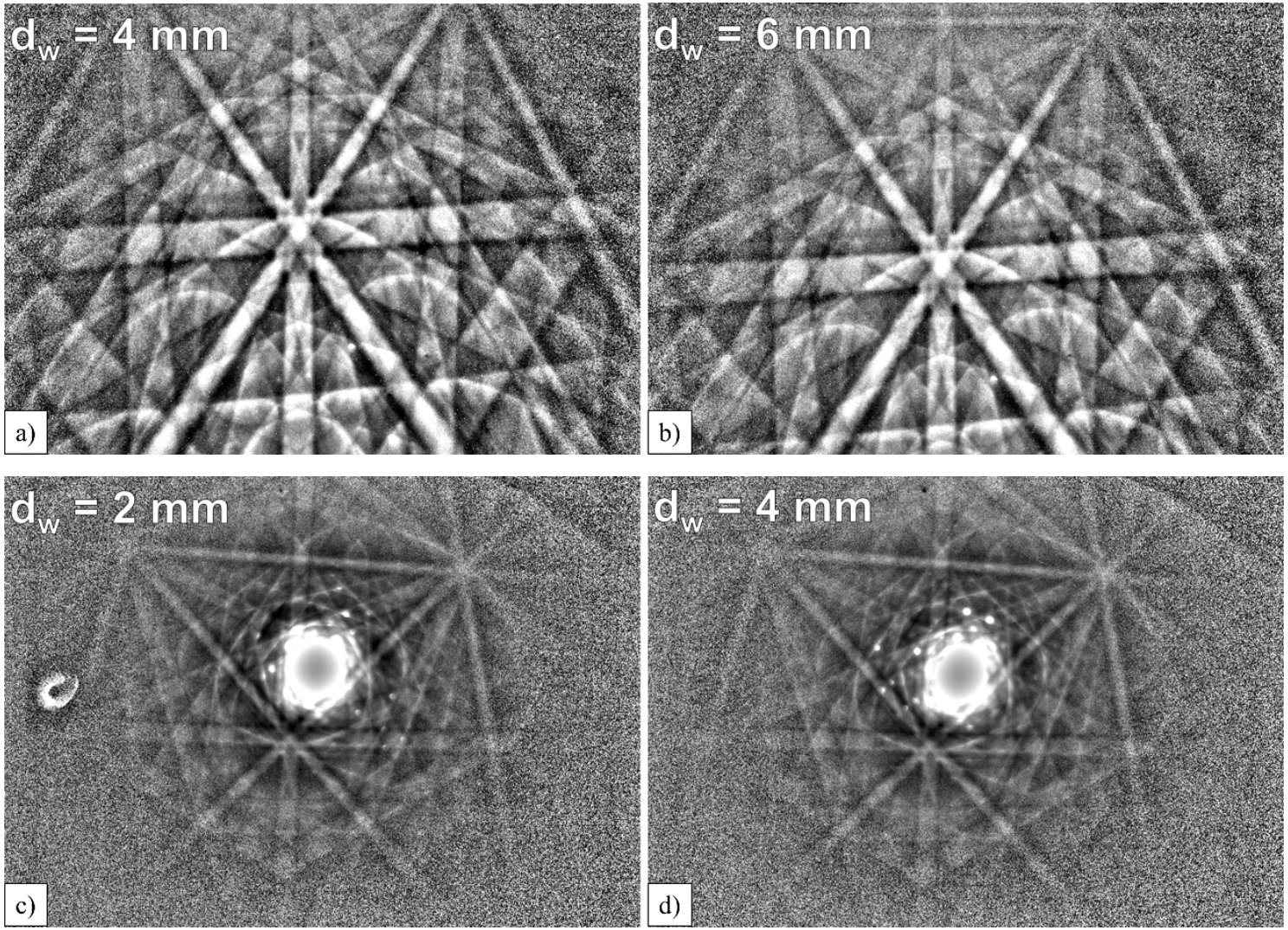

Figure 7 - Effect of working-distance, $d_{w}$, on Kikuchi patterns for (a-b): Off-axis TKD and a working distance of 4 and $6 \mathrm{~mm}$ in comparison to a working distance of 3mm shown in Figure 5a; (c-d): On-axis TKD and a working distance of 2 and $4 \mathrm{~mm}$ in comparison to a working distance of $6 \mathrm{~mm}$ shown in Figure 5d, for which the detectordistance was adapted to keep the distance between sample and detector constant

\section{Beam current}

The beam current was varied by changing the spotsize from 4 to 3.5, 4.5 and 5 . As different aperture settings were applied in on-axis and off-axis TKD (Table 2), the changes in spot size resulted in different beam-currents for both configurations. In off-axis TKD the beam current was correspondingly changed from $1.5 \mathrm{nA}$ to $0.74,3.00$ and $5.90 \mathrm{nA}$. The latter two beam current settings required a reduction of the exposure time to 500 and $200 \mathrm{~ms}$. In on-axis TKD the beam current was varied from $0.83 \mathrm{nA}$ to $0.42,1.70$ and $3.30 \mathrm{nA}$. In the latter two cases the exposure time needed to be reduced to 50 and 25 ms.

The pattern contrast generally increased with increasing beam current. In the case of the on-axis detector the increase in beam current on the one hand led to a larger area of the pattern which was disturbed by the transmitted beam, but on the other hand also to an increase in contrast at the periphery of the pattern (Figure 8). 

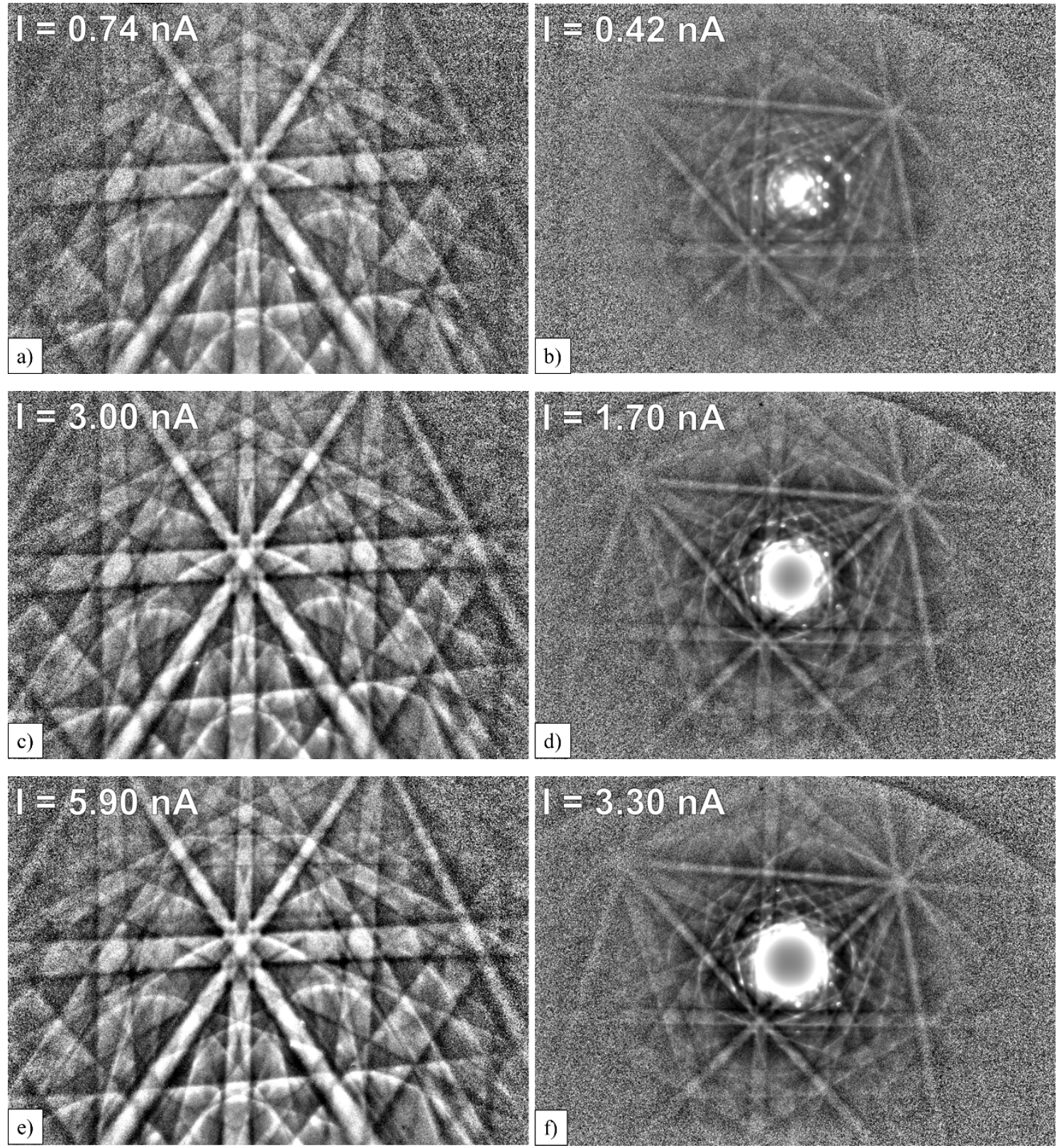

Figure 8 - Effect of beam current on Kikuchi patterns for (a,c and e): the off-axis detector-configuration and beam currents of $0.74,3.00$ and $5.90 \mathrm{nA}$, respectively, compared to $1.50 \mathrm{nA}$ in Figure $5 \mathrm{a}$; (b, $\mathrm{d}$ and e): the onaxis detector-configuration and beam currents $0.42,1.70$ and $3.30 \mathrm{nA}$, respectively, compared to $0.83 \mathrm{nA}$ in Figure 5d

\section{Beam energy}

Figure 9 shows the impact of lowering the beam energy from $30 \mathrm{keV}$ (Figure 5) to 25 and 20 $\mathrm{keV}$. In both off-axis and on-axis TKD the Kikuchi bands become less sharp and broaden with decreasing beam energy. In on-axis TKD it is evident that the size of the direct beam and the contrast of the spot-pattern gradually decrease with lower beam energy. 

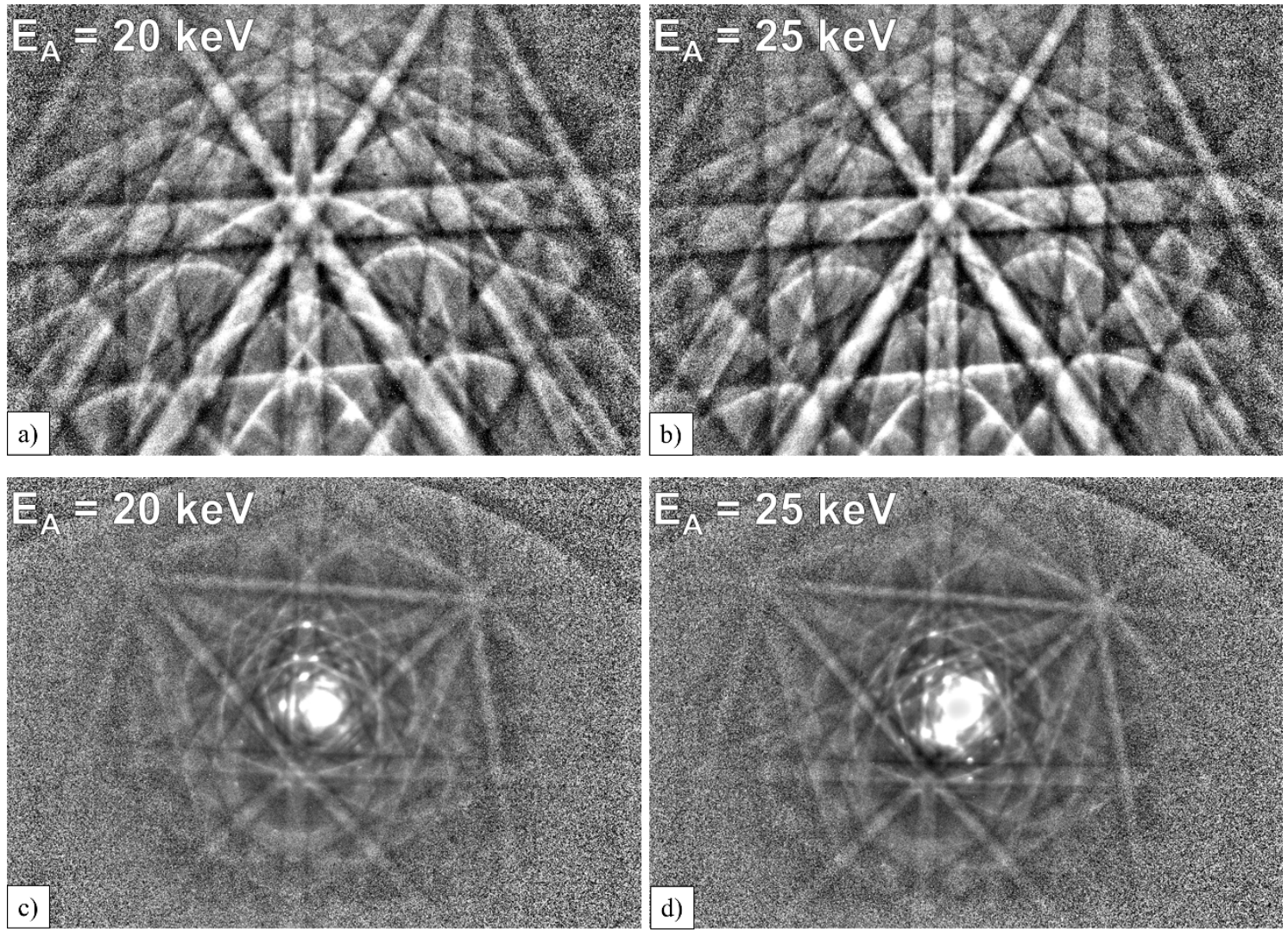

Figure 9 - Effect of beam energy on Kikuchi patterns for (a-b): the off-axis detector-configuration at 20 and 25 $\mathrm{keV}$ compared to $30 \mathrm{keV}$ in Figure 5a; (c-d): the on-axis detector-configuration at 20 and $25 \mathrm{keV}$ compared to 30 keV as shown in Figure $5 d$

\subsection{Kikuchi band detection and robustness of indexing}

Representative examples for the analysis of band detection and robustness of indexing are given in Figure 10a and b for off-axis TKD and in Figure 10c and d for on-axis TKD. Figure 10 a and c show the detected Kikuchi bands by Hough-transform (red lines) and the matching bands by the simulated pattern of the indexed phase and orientation (blue lines). Figure 10b and $\mathrm{d}$ show the indexed Kikuchi bands (blue lines) after manually refining the Kikuchi band positions (red lines).

Most obvious is the poor band detection by the Hough-transform algorithm in the lower right pattern region of the off-axis TKD pattern in Figure 10a. The algorithm seems to struggle with distinguishing excess and deficient lines from individual Kikuchi bands. In the presented case manual refinement of the band detection increased the amount of indexed bands from 8 to 10 (Figure 10b). The example for on-axis TKD (Figure $10 \mathrm{c}$ and d) demonstrates that manual refinement of band detection did not increase the number of indexed bands, which had already reached the software limit of 10 detectable bands. 

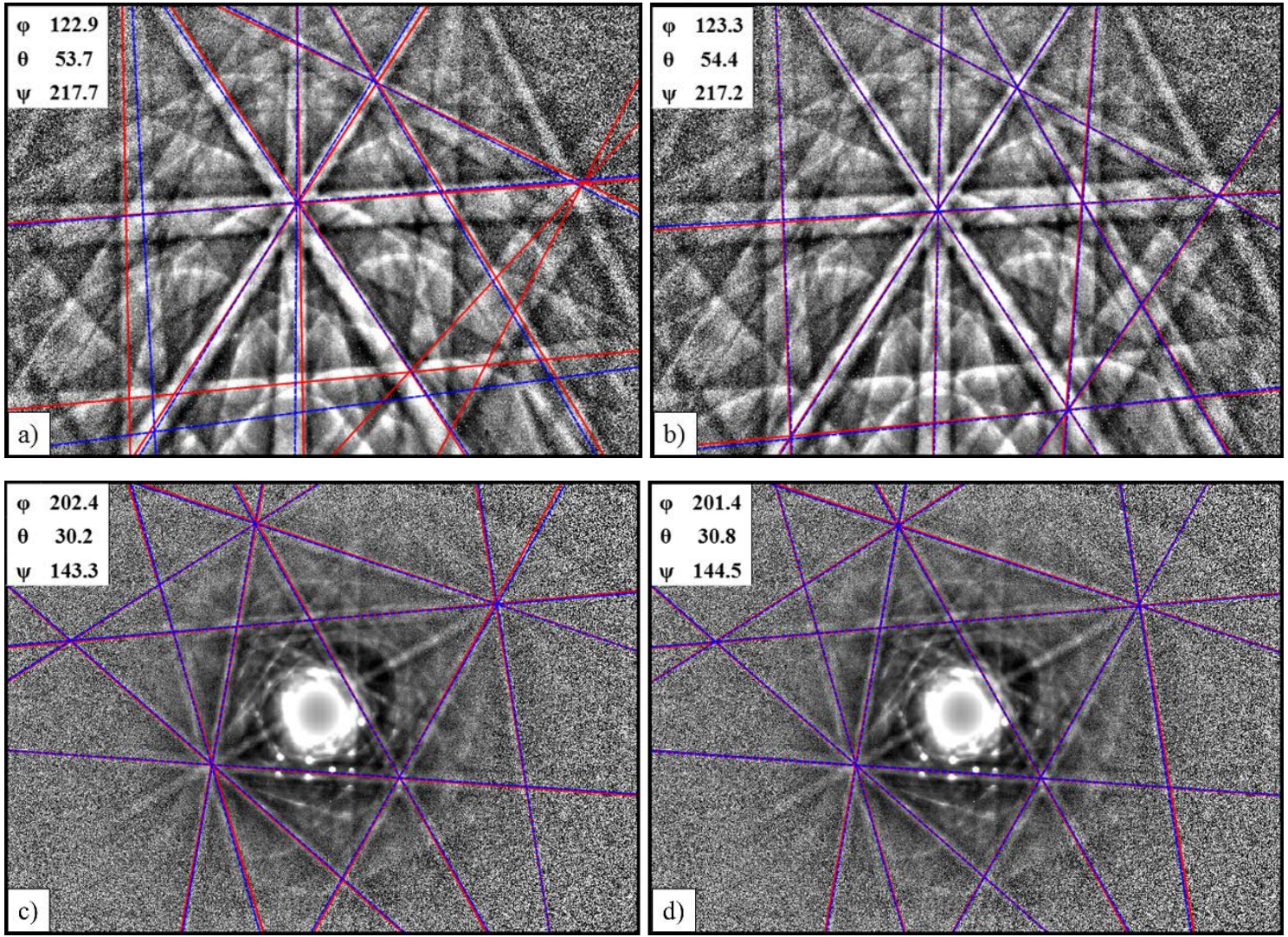

Figure 10 - Comparison of orientation-indexing by pattern simulation with Hough-transform based (a and c) and manual (b and d) line detection on off-axis (a and b) and on-axis (c and d) Kikuchi patterns. The detected and indexed Kikuchi bands are shown by red and blue lines, respectively. Gnomonic distortion leads to detection of Kikuchi lines as Kikuchi bands by the Hough-transform algorithm in off-axis TKD. In the example presented, manual refinement of the off-axis Kikuchi pattern led to two more indexed Kikuchi bands, whereas manual refinement of the on-axis Kikuchi pattern led to no additional indexed Kikuchi bands.

The presented examples were representative for the range of analyzed patterns. The average amount of indexed bands after band detection with the Hough-transform algorithm were $7.8 \pm 0.8$ and $8.8 \pm 1.3$ for off-axis and on-axis TKD, respectively. By manual refinement of the band detection the amount of indexed bands could be increased by an average of $1.6 \pm 0.4$ in off-axis TKD and was virtually unaffected in the case of on-axis TKD.

\subsection{Physical spatial resolution}

The physical spatial resolution (PSR) was determined by correlation of reference Kikuchi patterns to Kikuchi patterns across three different HAGBs. In on-axis TKD the working distance $d_{w}$ was altered to obtain measurements at the same working distance for both off-axis and on-axis TKD to exclude any influence of beam broadening. The results of the analysis are shown in Figure 11. The PSR of on-axis and off-axis TKD at $3 \mathrm{~mm}$ working distance was determined as $12.1 \pm 1.7 \mathrm{~nm}$ 


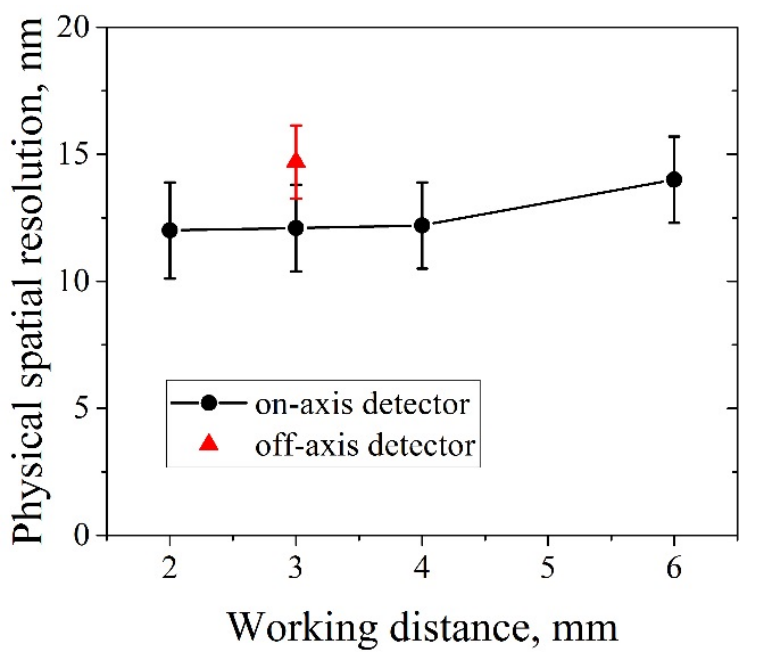

Figure 11 - PSR of the on-axis detector for different working distances in comparison to the PSR of the off-axis detector when applying the reference parameter sets (Table 2). The error-bars indicate the sample standard deviation from 30 different analyzed cropped pattern areas.

and $14.7 \pm 1.4 \mathrm{~nm}$, respectively. The PSR of on-axis TKD was not strongly affected by the working distance, only when increasing to a working distance of $6 \mathrm{~mm}$ a noticeable effect was visible.

\subsection{Effective spatial resolution (ESR)}

The effective spatial resolution (ESR) was analyzed by measuring a nanocrystalline gold thinfilm with the reference parameters from Table 2. To put the determined ESR into perspective, the physical spatial resolution on the thin-film was first determined in the same manner as for the austenitic stainless steel, but with $1 \mathrm{~nm}$ step size, and a step-size of 2 points for the moving average filter. The measurements were carried out on the biggest available grains, which were on the threshold of being to small to obtain good reference patterns. Consequently, the obtained values might be subject to inaccuracy and are given as an estimate, ranging between 7-8 $\mathrm{nm}$ in both onand off-axis TKD. All subsequently acquired orientation maps were acquired with $3 \mathrm{~nm}$ step size.

Because of drift issues in off-axis TKD when acquiring high-resolution patterns according to the reference parameters, the pattern resolution was reduced from $800 \times 600$ pixels to $320 \times 240$ pixels for both detector configurations in order to enable direct comparison over larger areas. Further reduction of the pattern resolution would have led to an additional mititgation of beam-drift, but led to overexposure of the on-axis TKD detector for the present sample and reference parameters. Figure 12a shows an overview map of the gold thin-film acquired with on-axis TKD. Unindexed points correspond to areas at which no information from diffraction could be obtained. Figure 12b to d show a magnification of a small area of different grain sizes, indicated by the rectangular area in Figure 12a. Figure 12b and Figure 12c show on-axis TKD maps acquired with pattern resolutions of 320 x 240 pixels and 800 x 600 pixels and exposure times of 12 and 75 ms, respectively. Figure 
12d shows the same area measured with off-axis TKD with a pattern resolution of $320 \times 240$ pixels and an exposure time of $136 \mathrm{~ms}$. The fraction of indexed points in on-axis TKD is clearly improved by measuring at better pattern resolution. Off-axis TKD reveals a high fraction of indexed points, but struggles with drift issues due to long measurement times even at lower pattern resolution. All tested conditions allowed indexing of grains with diameters of approx. $10 \mathrm{~nm}$, where the effective resolution at different GB's seemed to depend on the local quality of the sample.

Wherever sample quality was not an issue, the number of non-indexed points at grain boundaries were either 0 or 1 , corresponding to an effective resolution of equal or better to $3 \mathrm{~nm}$ in all three investigated conditions. Figure $12 \mathrm{~b}$ to $\mathrm{d}$ show that, in the left of the two large grains, a strongly

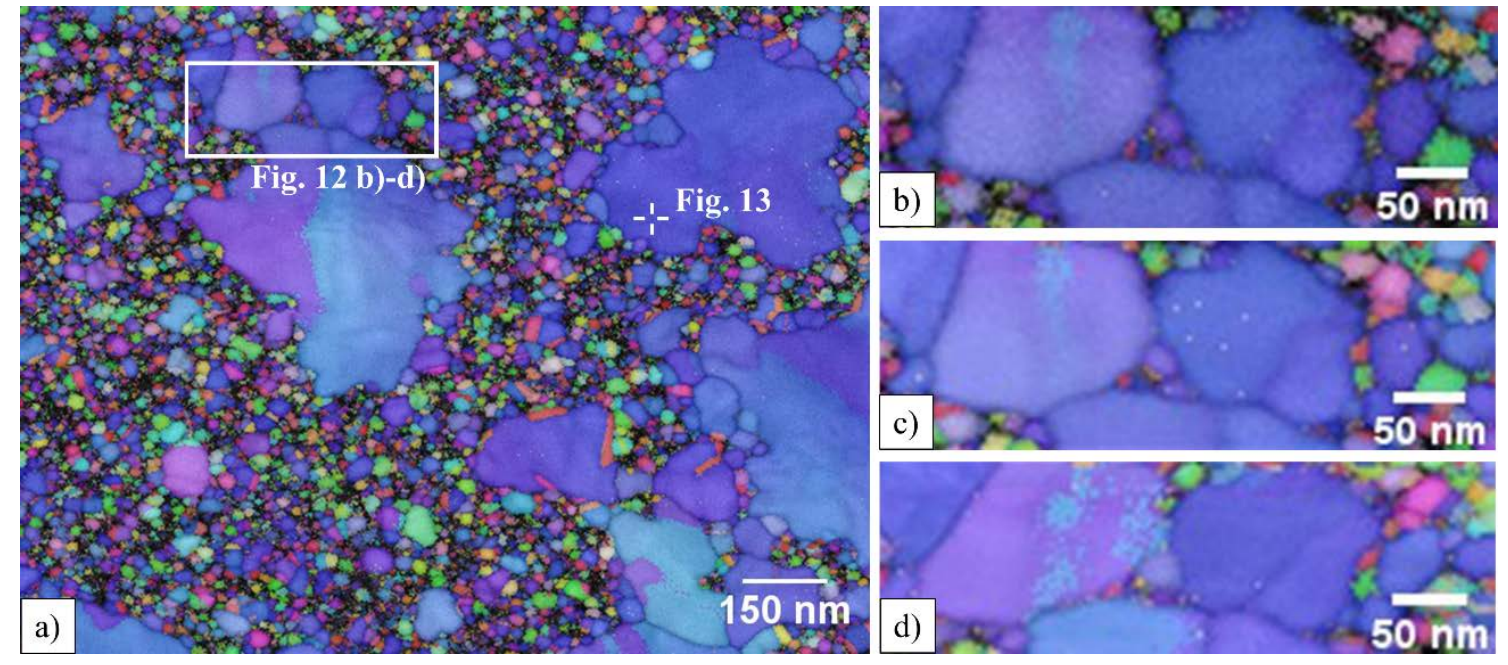

Figure 12 - Analysis of ESR on inverse pole-figure map of nanocrystalline gold thin-film; if not stated differently the reference parameters in Table 2 are applied; a) On-axis TKD: Overview map acquired with a reduce pattern resolution of $320 \times 240$ pixels and $12 \mathrm{~ms}$ exposure time; b) On-axis TKD: Inset of the region marked with a rectangle in Figure 12a) acquired with identical parameters; c) On-axis TKD: Scan on the same inset with $800 \mathrm{x}$ 600 pixels pattern resolution and 75 ms exposure time; d) Off-axis TKD: Scan of the inset with 320 x 240 pixels pattern resolution and $136 \mathrm{~ms}$ exposure time

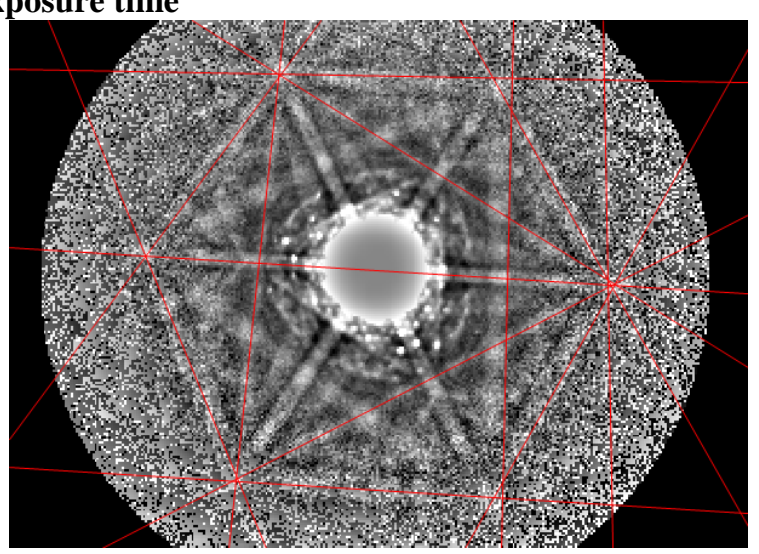

Figure 13 - Kikuchi pattern and detected bands (red lines) from the spot marked in Figure $12 a$, obtained at 320 x 240 pixels pattern resolution and $12 \mathrm{~ms}$ exposure time. On-axis Kikuchi patterns are indexed robustly even when the direct beam overlaps with a major zone axis. The major diffraction information then arises mainly from surrounding zone axes 
inclined twin boundary was indexed differently by on-axis and off-axis TKD. Closer analysis revealed that an overlap of the parent and twin orientations in both on-axis and off-axis TKD patterns led to ambiguous indexing. As indexing of the twin was also slightly affected by changing the pattern resolution in on-axis TKD with otherwise unaltered parameters (Figure 12b and c), the difference in indexing in on-axis and off-axis TKD is likely to depend on detection of characteristic bands of either orientation by the Hough transform, and may to a lesser extent be an effect of different specimen tilt or source region. It is important to point out that contamination of the mapped area was quite significant and the repeated measurements on the same area were influenced by it. First the off-axis measurement was performed (Figure 12d), followed by the high resolution on-axis map (Figure 12c) and finally the lower resolution map (Figure 12b).

Figure 13 shows the Kikuchi pattern which was obtained with on-axis TKD from the marked position in Figure 12a. The pattern is representative for patterns in which the major zone axis is obscured by the bright spot caused by the transmitted beam. In such cases robust indexing was still possible, mainly based on surrounding zone axes, and in some cases even supported by bands that cross the spot of the direct beam.

\section{Discussion}

It was generally observed that on-axis Kikuchi patterns, because of the different detector geometry, are less sensitive to changes in working distance and detector distance. Moreover, onaxis Kikuchi patterns were more affected by changes in beam current and beam energy, as the signal is acquired at the maximum intensity of the Kikuchi cone and also contains the direct beam and coherently scattered electrons. This general observation, its consequences for orientation mapping, and related observations are discussed in further detail in the following section.

\subsection{Kikuchi pattern area}

By comparing the reference Kikuchi patterns with their simulated patterns in Figure 5 it was found that, although similar detector distances were chosen, the capture angle in on-axis TKD was much larger, which resulted in approximately $70 \%$ more area of the Kikuchi map. This area is obviously dependent on the chosen detector distance, which in on-axis TKD can be tuned over a wide range without losing signal intensity, as demonstrated in Figure 6c and d. The limited interception with the Kikuchi cone in off-axis TKD leads to severe loss of intensity and detectable bands with increasing detector distance, which makes it a rather sensitive parameter (Figure 6a and 
b). The adaptability of the on-axis setup opens a wide range of applications, ranging from general orientation mapping (short detector distance) to strain mapping (large detector distance).

A different way of changing the effective Kikuchi pattern area in on-axis TKD is by adjustment of the beam current (Figure 8b, d and f). While a lower beam current naturally improves the PSR, the ESR is compromised by the decrease in pattern intensity. Thus sacrificing PSR by measuring with higher beam current can, in fact, lead to a considerable improvement in ESR by obtaining more detectable Kikuchi bands (see Figure 8). Furthermore, acquisition rate, and thus sensitivity to drift, as well as indexing robustness are improved with higher beam current, as reported for off-axis TKD in Ref. [4]. As a downside, increased beam-current leads to increased contamination. Finally, the effect of the transmitted beam leads to a sample dependent upper limit for the beam current.

\subsection{Working distance}

Generally, shorter working distance is favorable as it minimizes the effect of beam broadening. In off-axis TKD the working distance is critical for obtaining good orientation mapping, as the signal intensity and the pattern center are directly affected by this parameter (Figure 7a and b). For a given working distance, the detector distance should be minimized and the detector tilt should be adapted for optimal illumination of the phosphor screen. With decreasing working distance the detector is tilted increasingly upwards to obtain optimum signal. This leads to an effective increase of the intersection angle, $\alpha$, and consequently to increased intensity and reduced gnomonic distortion. When using a detector that operates at fixed tilt angle, low working distance may, in contrast, lead to analysis far away from the pattern center and thus lead to stronger gnomonic distortion.

In on-axis TKD the working distance had no significant influence on the Kikuchi pattern (Figure 7c and d), although at working distances larger than $6 \mathrm{~mm}$ an evident decrease in PSR was observed (Figure 11), which is most likely caused by beam broadening.

\subsection{Pattern contrast}

Decreasing the beam energy led, in both on- and off-axis TKD, to a decrease in pattern contrast and to broadening of the Kikuchi bands, as generally expected according to the physics of Kikuchi pattern formation [16] and specifically reported for TKD in Refs. [5,7,12,17,18]. In on-axis TKD, lowering the beam energy furthermore led to a reduction in the area of the transmitted beam and lower intensity of the diffraction spots. In Ref. [7] the loss of plasmon and phonon scattering with 
decreasing beam energy is found as the main reason for this effect. As diffraction spots are not desired for standard TKD-investigations, the beam energy can be used to balance the contrast when working with very thin specimens or specimens of low atomic number.

Increasing the beam current led to sharpening of the Kikuchi lines in both detector configurations, as also reported in [19]. Generally the beam current can therefore be adapted to gain pattern contrast, in which case the aforementioned effects on the intensity of the transmitted beam, the effective Kikuchi pattern area and the PSR should be considered.

\subsection{Kikuchi band detection and robustness of indexing}

Comparison of Figure 5c and f shows that the Hough space of the on-axis Kikuchi pattern is not noticeably affected by the bright spot caused by the transmitted beam. The robustness to noise and discontinuities in the patterns is in fact the strength of the Hough transform [20,21]. Figure 13 shows that, even though the major zone axis of the pattern coincided with the bright spot, robust indexing was possible, although mainly by surrounding zones axes in the periphery of the pattern. Sneddon et al. speculated, that the bright spot from the direct beam may cause issues with indexing relatively indistinct patterns [8]. In cases, where only one zone axis is present and this coincides with the bright spot, indexing can in fact be compromised. Whenever such a situation is observed, decreasing detector distance (by either changing working distance and/or detector tilt) improves indexing. In fact, off-axis patterns of such orientations were found to be more problematic, as unambiguous indexing based on a single zone axis was difficult, and changing the detector distance was unfeasible. Because of the large available area of the Kikuchi map and the consequently high amount of detectable bands, on-axis TKD is particularly well suited for phase identification.

The robustness of Kikuchi band detection and indexing of both on-axis and off-axis Kikuchi patterns were analyzed by comparing pattern indexing based on band detection by the Houghtransform and by manual refinement (Figure 10). The fact that manual band detection improves indexing robustness more significantly in off-axis rather than in on-axis TKD, indicates that the gnomonic distortion is more detrimental for the Hough based indexing routine than the bright area of the transmitted beam in the pattern center. Gnomonic distortion led to strong magnification of excess and deficient lines of Kikuchi bands in the lower pattern region, which were detected as individual Kikuchi bands by the Hough transform (c.f. Figure 10c). This issue was previously reported in Ref. [4], and is virtually absent in on-axis TKD. Some commercially available indexing softwares have made efforts to partially mitigate this effect in off-axis TKD by modifiying the band 
detection algorithm to account for this change in geometry [8]. Such software correction was not applied in the present work so that its effectiveness could not be evaluated.

\subsection{Acquisition rate}

One of the most striking advantages of on-axis TKD compared to off-axis TKD was the immense gain in acquisition rate. The reference measurements with on-axis and off-axis TKD were conducted with $0.83 \mathrm{nA}$ and $1.5 \mathrm{nA}$ beam current, respectively. The product of the beam current ratio with the applied exposure time ratio resulted in a factor of 20 when measuring with the reference parameters from Table 2 or with lower pattern resolution and exposure time as in Figure 12. This observation confirms the findings by Yuan et al., who reported that for a fixed incident intensity on the specimen in comparison to off-axis TKD the acquisition time is at least 20 times shorter at equivalent pattern quality and indexation rate, and that alternatively 20 times lower electron dose can be used [6].

Such an increase in acquisition rate may be beneficial to save microscope time, but is of far greater importance to utilize the new possibilities, which TKD gives in terms of spatial resolution. A clear distortion of the orientation map caused by beam drift is evident in the orientation map from off-axis TKD in Figure 12d, when comparing the equivalent measurements from on-axis TKD in Figure $12 \mathrm{~b}$ and $\mathrm{c}$. The issue of beam drift in conventional TKD was also observed and discussed in Refs. $[6,22,23]$. In the present case, further binning of the Kikuchi pattern in off-axis TKD would have mitigated the extensive beam drift to some extent, but would have led to overexposure of the camera in on-axis TKD, when testing in identical conditions. Even though beam drift can be reduced to some extent by letting the sample settle for a few hours in the evacuated microscope chamber or by installing an advanced cooling system of the lens coils, it will remain an issue in both on- and off-axis TKD. The increase in acquisition rate gained in on-axis TKD is therefore important to acquire large data sets with minimum beam drift, and thus for utilizing the reported spatial resolutions in practice. In cases were both on-axis and off-axis TKD are capable of running at acquisition rates that are limited by the detector hardware, on-axis TKD can be operated with higher pattern resolution or lower beam current.

\subsection{Spatial resolution}

On-axis TKD led to a small improvement of 2-3 nm in PSR in measurements over HABG's on a $115 \pm 8 \mathrm{~nm}$ thick austenitic stainless steel thin-foil (Figure 11) with the stated sets of reference 
parameters in Table 2. The measured difference in PSR is seen representative for measuring with detector-typical beam energy, beam current and specimen tilt and may furthermore be affected by the source region of the acquired intensity.

On-axis and off-axis detector systems acquire the intensity from electrons of different scattering angle (Figure 1). Considering a given average path length of an electron from the last incoherent scattering event to leaving the exit surface of the sample, the increased scattering angle of electrons detected in off-axis TKD may lead to a thinner source region of the detected intensity, as suggested in Ref. [8]. Assuming that such effect is noticeable, it would for a given sample thickness imply better depth resolution, but worse lateral resolution of off-axis TKD compared to on-axis TKD. The source region effect may partially be responsible for the slightly better PSR of on-axis TKD in the present case (Figure 11), but dedicated investigations on bilayered samples are required to obtain a better understanding.

Lower specimen tilt in off-axis TKD could potentially lead to an improvement of the measured values, but led in the present case to insufficient intensity at the upper part of the phosphor screen. Ref. [24] suggests a worsening of the lateral PSR with increasing specimen backtilt without providing a quantitative analysis, which to the best knowledge of the authors has neither been reported elsewhere.

The applied exposure times in this spatial resolution study, especially for off-axis TKD, result in too low acquisition rates to be considered for investigating larger areas. In order to increase the acquisition rate for a large area scan the options would be to increase the beam current, which compromises the PSR, or reducing the pattern resolution, which compromises the ESR. Therefore, the spatial resolution in off-axis TKD is expected to be worse when measuring larger maps, while the same is not the case for on-axis TKD, which intrinsically operates at higher acquisition rates (see discussion in section 4.5).

The ESR was estimated from measurements on a nanocrystalline gold thin-film (Figure 12). The physical spatial resolution was estimated to range between 7-8 $\mathrm{nm}$ and it was observed that both detector configurations managed to resolve grains of approx. $10 \mathrm{~nm}$ in diameter. The on-axis TKD scans led to noticeably better indexing when increasing the pattern resolution from $320 \times 240$ pixels to $800 \times 600$ pixels at the cost of measuring at $16 \%$ of the original acquisition rate. The detector system is advertised by the manufacturer to enable orientation mapping with an ESR of $3 \mathrm{~nm}$ or better on a variety of tested materials. On thin samples of high atomic number, which are optimal for achieving high spatial resolution, this figure is regarded as realistic, as similar ESR could be 
obtained when measuring the $15 \mathrm{~nm}$ thick nanocrystalline gold thin-film. A measurement performed with $1 \mathrm{~nm}$ step size and pattern resolution of 320x240 revealed 1-2 non indexing points at the grain boundaries, indicating that the effective resolution can be even better.

\subsection{General remarks and outlook}

Most conventional EBDS detectors contain a set of diodes designed for forward scatter imaging, which are well-suited to conduct dark-field imaging in off-axis TKD [22]. The geometry of the onaxis detector configuration in turn enables continuous switching between true bright- and dark-field contrast similar to imaging in TEM (c.f. bright-field image in Figure 3a) by inserting the detector to different insertion distances. As TKD, thanks to its good spatial resolution, is generally well suited for characterization of ultrafine grained microstructures with high defect density [6,22], these imaging capabilities qualify the on-axis detector configuration even further for such application.

In order to switch between different imaging modes and the TKD mode, the insertion distance of the on-axis detector has to be changed to allow acquisition of the transmitted intensity with the diodes or the phosphor screen, respectively. A drawback of changing between acquisition modes in this way is that movement of the detector leads to deflection of the electron beam, which becomes more prominent with increasing working distance. This effect neither influences the quality of Kikuchi patterns nor the measurement performance, but may cause difficulties in locating the same position after imaging on very fine microstructures. Such issue is not as pronounced in the off-axis configuration. Even though imaging at increased detector distance also leads to better image contrast when using the diodes on the EBSD-detector, it is possible to both obtain acceptable image quality and good orientation maps at a single detector distance.

Simultaneous EDS and TKD analysis are possible in the on-axis geometry, but lead to a strong $\mathrm{X}$-ray fluorescence signal from the interaction of the direct beam with the detector when using the standard configuration presented in this work [19]. It was found that this obstructive effect can be largely supressed by using a purpose-fit stage to shield the signal of the on-axis detector. Further, some recently developed silicon drift detectors for EDS analysis [25,26] can largely mitigate this issue, as they are inserted between the pole piece and the electron transparent sample to provide a large solid angle. The increased signal yield allows simultaneous EDS and TKD analysis at high acquisition rate [27], reducing the currently experienced drift issues when using conventional hardware . 
Brodu et al. found that the contrast of on-axis TKD patterns is sensitive to changes in thickness and beam energy [7]. Generally, more distinct features, such as diffraction spots, Kikuchi bands and Kikuchi lines, become visible as a function of these parameters. This change in contrast was modeled by a simple equation, which enables tuning it for specific materials. Even though the effect of sample thickness was not part of the present investigation, no dramatic change in patter contrast or indexing precision were experienced when mapping areas on steel samples with several $10 \mathrm{~nm}$ deviation in thickness. For samples with extreme deviation in thickness off-axis TKD could lead to better indexing, as the contrast of off-axis patterns was found less sensitive to changes in thickness.

As the development of the on-axis detector is still at an early stage, there is a need to develop software which employs the variety of features contained in an on-axis Kikuchi pattern in a more distinct way [7]. Currently, for instance the band contrast map is disturbed by acquisition of the transmitted beam, which might be corrected by omitting the overexposed area in the center of the pattern. It was reported in Ref. [7] that specimen thickness and incident energy have a symmetrical influence on the diffraction contrast in on-axis TKD. If this relation holds, the bright area of the transmitted beam, which is a measurement of electron absorption, could be used to estimate the specimen thickness when the beam energy, beam current and atomic number of the specimen are known. In this way on-axis TKD may in parallel to conventional orientation mapping enable acquisition of thickness maps similar to those obtained from EELS in TEM. For such an application further understanding of the diffraction contrast and in particular the influence of crystal orientation on the absorption of the transmitted beam is needed.

\section{Conclusions}

- On-axis TKD was found advantageous over off-axis TKD mainly due to the evident enhanced pattern intensity, which allows reduction of the beam current and/or increasing the acquisition rate, and due to virtual absence of gnomonic distortion. Fast acquisition over large areas enabled considerable reduction of drift issues.

- The measured PSR obtained using detector-typical microscope parameters reveals a relatively small improvement of using on-axis TKD. To measure the PSR at the same pattern resolution, long exposure times were required in off-axis TKD. In larger area scans the spatial resolution of off-axis TKD is therefore expected to be worse, since an increase in beam current or decrease in pattern resolution is required. 
- As a measurement of the ESR gold grains of approximately $10 \mathrm{~nm}$ could be reliably indexed by both on-axis and off-axis TKD. In off-axis TKD the achieved resolution was mitigated by beam drift.

- On-axis TKD, based on its intersection with the intensity maximum of the Kikuchi cone below the specimen, is less sensitive to changes in geometry (working distance and detector distance) but more sensitive to changes in beam current and beam energy than off-axis TKD.

- Hough transform based automated band detection led to on average one more indexed band per pattern in on-axis TKD compared to off-axis TKD. Manual refinement of band detection led to indexing of on average 1.6 additional bands in off-axis and virtually no further increase in on-axis TKD. Consequently the Hough transform seems to operate more robustly on on-axis TKD patterns.

- The bright spot caused by the transmitted beam in on-axis TKD did not noticeably disturb the Hough transform. In the case where the major zone axis coincided with the bright spot of the transmitted beam, additional zone axes in the periphery of the Kikuchi pattern could be detected.

\section{Acknowledgement}

Jens Kling, DTU Center for Electron Nanoscopy (CEN), is acknowledged for conducting the thickness measurement on the austenitic stainless steel thin foil. Bruker Nano GmbH (BNA) is gratefully acknowledged for the loan of the OPTIMUS ${ }^{\mathrm{TM}}$ TKD detector head. The Danish Underground Consortium is gratefully acknowledged for financial support to the Danish Hydrocarbon Research Center (DHRTC).

\section{References}

[1] R.H. Geiss, R.R. Keller, D.T. Read, Transmission electron diffraction from nanoparticles, nanowires and thin films in an SEM with conventional EBSD equipment, Microsopy Microanal. 16 (2010) 1742-1743.

[2] R. Geiss, R. Keller, S. Sitzman, P. Rice, New Method of Transmission Electron Diffraction to Characterize Nanomaterials in the SEM, Microsc. Microanal. 17 (2011) 386-387. 
[3] R.R. Keller, R.H. Geiss, Transmission EBSD from $10 \mathrm{~nm}$ domains in a scanning electron microscope, J. Microsc. 245 (2012) 245-251.

[4] P.W. Trimby, Orientation mapping of nanostructured materials using transmission Kikuchi diffraction in the scanning electron microscope, Ultramicroscopy. 120 (2012) 16-24.

[5] J.J. Fundenberger, E. Bouzy, D. Goran, J. Guyon, H. Yuan, A. Morawiec, Orientation mapping by transmission-SEM with an on-axis detector, Ultramicroscopy. 161 (2016) 17-22.

[6] H. Yuan, E. Brodu, C. Chen, E. Bouzy, J.-J. Fundenberger, L.S. Toth, On-axis versus offaxis Transmission Kikuchi Diffraction technique: application to the characterisation of severe plastic deformation-induced ultrafine-grained microstructures, J. Microsc. 0 (2017) 1-11.

[7] E. Brodu, E. Bouzy, J.-J. Fundenberger, Diffraction contrast dependence on sample thickness, incident energy and atomic number in on-axis Transmission Kikuchi Diffraction in the SEM, Ultramicroscopy. 181 (2017) 123-133.

[8] G.C. Sneddon, P.W. Trimby, J.M. Cairney, Transmission Kikuchi diffraction in a scanning electron microscope: A review, Mater. Sci. Eng. R Reports. 110 (2016) 1-12.

[9] S. Zaefferer, On the formation mechanisms, spatial resolution and intensity of backscatter Kikuchi patterns, Ultramicroscopy. 107 (2007) 254-266.

[10]T. Malis, S.C. Cheng, R.F. Egerton, Log-Ratio Technique for Specimen-Thickness Measurement in the TEM, J. Electron Microsc. Tech. 8 (1988) 193-200.

[11]D.R.G. Mitchell, B. Schaffer, Scripting-customised microscopy tools for Digital Micrograph(TM), Ultramicroscopy. 103 (2005) 319-332.

[12]K.P. Rice, R.R. Keller, M.P. Stoykovich, Specimen-thickness effects on transmission Kikuchi patterns in the scanning electron microscope, J. Microsc. 254 (2014) 129-136.

[13]D. Chen, J.C. Kuo, W.T. Wu, Effect of microscopic parameters on EBSD spatial resolution, Ultramicroscopy. 111 (2011) 1488-1494.

[14]Y.Z. Wang, M.G. Kong, Z.W. Liu, C.C. Lin, Y. Zeng, Effect of microscope parameter and specimen thickness of spatial resolution of transmission electron backscatter diffraction, J. Microsc. 264 (2016) 34-40.

[15] MATLAB 2015a - Imaging Toolbox - function corr2, (2015).

[16]D.B.B. Williams, C.B. Carter, C. Barry Carter, Transmission Electron Microscopy, 2nd ed., Springer, 2009. 
[17]J.-W. Shih, K.-W. Kuo, J.-C. Kuo, T.-Y. Kuo, Effects of Accelerating Voltage and Specimen Thickness on the Spatial Resolution of Transmission Electron Backscatter Diffraction in Cu, Ultramicroscopy. 177 (2017) 43-52.

[18] S. Suzuki, Features of transmission EBSD and its application, JOM. 65 (2013) 1254-1263.

[19] M. Abbasi, D. Kim, H. Guim, M. Hosseini, H. Danesh-manesh, M. Abbasi, Application of Transmitted Kikuchi Diffraction in Studying Nano-oxide and Ultrafine Metallic Grains, ACS Nano. 9 (2015) 10991-11002.

[20]F. Karsli, O. Kahya, Building extraction from laser scanning data, Int. Arch. Photogramm. Remote Sens. Spat. Inf. Sci. XXXVII (2008) 289-294.

[21]N.C. Krieger Lassen, D. Juul Jensen, K. Conradsen, Image processing procedures for analysis of electron back scattering patterns, Scanning Microscoy. 6 (1992) 115-121.

[22]P.W. Trimby, Y. Cao, Z. Chen, S. Han, K.J. Hemker, J. Lian, X. Liao, P. Rottmann, S. Samudrala, J. Sun, J.T. Wang, J. Wheeler, J.M. Cairney, Characterizing deformed ultrafine-grained and nanocrystalline materials using transmission Kikuchi diffraction in a scanning electron microscope, Acta Mater. 62 (2014) 69-80.

[23]N. Brodusch, H. Demers, R. Gauvin, Nanometres-resolution Kikuchi patterns from materials science specimens with transmission electron forward scatter diffraction in the scanning electron microscope, J. Microsc. 250 (2013) 1-14.

[24]G. Sneddon, P. Trimby, J. Cairney, The Influence of Microscope and Specimen Parameters on the Spatial Resolution of Transmission Kikuchi Diffraction, Microsc. Microanal. 23 (2017) 532533.

[25]H. Soltau, A. Bechtele, O. Jaratschin, A. Liebl, A. Niculae, A. Simsek, R. Eckhard, K. Hermenau, P. Lechner, G. Lutz, G. Schaller, F. Schopper, L. Strüder, SDDs - Silicon Detectors for EDS with a Performance at the Theoretical Limit, Microsc. Microanal. 14 (2008) 1156-1157.

[26]A. Niculae, H. Soltau, P. Lechner, A. Liebl, G. Lutz, L. Strüder, R. Eckhard, G. Schaller, F. Schopper, Optimum Performance for Microanalysis with Silicon Drift Detectors with Integrated FET, Microsc. Microanal. 13 (2007) 1430-1431.

[27]D. Goran, Quantitative Characterization of Nanostructured Materials with Fast TKD Measurement - Bruker Webinar, (2017). 